\title{
Norm of matrix operator on Orlicz-binomial spaces and re- lated operator ideal
}

\author{
Taja Yaying ${ }^{\mathrm{a}}$, Bipan Hazarika ${ }^{\mathrm{b}, *}$, M. Mursaleen ${ }^{\mathrm{c}, \mathrm{d}}$ \\ ${ }^{a}$ Department of Mathematics, Dera Natung Govt. College, Itanagar 791111, Arunachal Pradesh, India. \\ ${ }^{b}$ Department of Mathematics, Gauhati University, Guwahati 781014, Assam, India. \\ ${ }^{c}$ Department of Marthematics, Aligarh Muslim University, Aligarh 202002, India. \\ ${ }^{d}$ Department of Medical Research, China Medical University Hospital, China Medical University (Taiwan), Taichung, Taiwan.
}

\begin{abstract}
The purpose of this article is to introduce Orlicz extension of binomial sequence spaces $\mathbf{b}_{\varphi}^{r, s}$ and investigate some topological

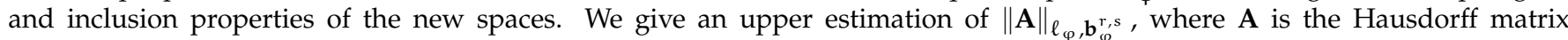
operator or Nörlund matrix operator. A Hardy type formula is established in the case of Hausdorff matrix operator. Finally we introduce operator ideal using the space $\mathbf{b}_{\varphi}^{r, s}$ and the sequence of s-number function and prove its completeness under certain assumptions.
\end{abstract}

Keywords: Binomial sequence space, upper bounds, Hausdorff Matrix, Nörlund Matrix, Orlicz function, s-number, operator ideal.

2020 MSC: 26D15, 46A45, 47A30, 40G05, 47L20, 47B06.

(C)2021 All rights reserved.

\section{Introduction}

Throughout this paper $\mathbb{N}_{0}=\{0,1,2, \ldots\}$ and $\omega$ denotes the linear space of all real sequences. Any vector subspace of $\omega$ is called a sequence space. Also by $\ell_{p}$, we mean the space of absolutely $p$-summable series, where $1 \leqslant p<\infty$. The space $\ell_{p}$ is a Banach space according to the $\ell_{p}$ norm given by

$$
\|\mathbf{x}\|_{p}=\left(\sum_{k=0}^{\infty}\left|x_{k}\right|^{p}\right)^{\frac{1}{p}} .
$$

For some recent papers on sequence spaces, one may refer to $[5,8,16,20,36,39,40]$. Let $X$ and $Y$ be two sequence spaces and let $\mathbf{A}=\left(a_{n k}\right)$ be an infinite matrix of real entries. We write $\mathbf{A}_{n}$ to denote the sequences in the $n$th row of the matrix $\mathbf{A}$. We recall that $\mathbf{A}$ defines a matrix mapping from $X$ to $Y$ if for

\footnotetext{
*Corresponding author

Email addresses: tajayaying20@gmail.com (Taja Yaying), bh_rgu@yahoo.co.in; bh_gu@gauhati.ac.in (Bipan Hazarika), mursaleenm@gmail.com (M. Mursaleen)
}

doi: $10.22436 /$ jmcs.023.04.07

Received: 2020-02-11 Revised: 2020-03-29 Accepted: 2020-04-08 
every sequence $\mathbf{x}=\left(x_{k}\right)$, the A-transform of $\mathbf{x}$, i.e., $\mathbf{A x}=\left\{\mathbf{A}_{\mathfrak{n}} \mathbf{x}\right\}_{\mathfrak{n}=0}^{\infty} \in \mathrm{Y}$, where

$$
\mathbf{A}_{n} \mathbf{x}=\sum_{k=0}^{\infty} a_{n k} x_{k}, \quad n \in \mathbb{N}_{0} .
$$

An Orlicz function $\varphi$ is a function from $(0, \infty)$ to $(0, \infty)$ which is continuous, increasing and convex with $\varphi(0+)=0$ and so has a unique inverse $\varphi^{-1}:(0, \infty) \rightarrow(0, \infty)$. As usual in the Orlicz theory the domain of $\varphi$ is extended to the real line by $\varphi(x)=\varphi(|x|)$ and $\varphi(0)=0$ (for details on Orlicz functions see [12, 13, 27]).

Let $\mathbf{x}=\left(x_{n}\right)$ be a sequence of real numbers with $x_{n}>0$ for all $n \in \mathbb{N}_{0}$. The Orlicz sequence space is defined as

$$
\ell_{\varphi}=\left\{x \in \omega: \sum_{n=0}^{\infty} \varphi\left(\frac{x_{n}}{\rho}\right)<\infty \text { for some } \rho>0\right\} .
$$

The space $\ell_{\varphi}$ is a Banach space equipped with the Orlicz-Luxemborg norm $\|\cdot\|_{\varphi}$, defined by

$$
\|x\|_{\varphi}=\inf \left\{\rho>0: \sum_{n=0}^{\infty} \varphi\left(\frac{x_{n}}{\rho}\right) \leqslant 1\right\} .
$$

Clearly $\left|x_{n}\right| \leqslant\left|y_{n}\right|$ for all $n \in \mathbb{N}_{0}$, then $\|\mathbf{x}\|_{\varphi} \leqslant\|\mathbf{y}\|_{\varphi}$. Further if $0<\|\mathbf{x}\|_{\varphi}<\infty$, then $\sum_{n=0}^{\infty} \varphi\left(\frac{x_{n}}{\|\mathbf{x}\|_{\varphi}}\right) \leqslant 1$ [25, Lemma 1].

In particular, if $\varphi(t)=|t|^{p}, p \geqslant 1$, then the space $\ell_{\varphi}$ reduces to the $\ell_{p}$ space and the norm $\|\mathbf{x}\|_{\varphi}$ given by (1.2) reduces to the norm $\|\mathbf{x}\|_{p}$ given by (1.1).

By supermultiplicative function, we shall mean any function $\varphi:(0, \infty) \rightarrow(0, \infty)$ such that for all positive $a$ and $b$

$$
\varphi(a b) \geqslant \varphi(a) \varphi(b)
$$

An immediate example of supermultiplicative function is $\varphi(t)=t^{p}, p \geqslant 1$. Throughout the article, we consider this supermultiplicative Orlicz function $\varphi$ which satisfies $\varphi(1)=1$.

We recall that an upper bound for a matrix operator $\mathcal{T}$ from a sequence space $X$ into another sequence space $\mathrm{Y}$ is the value of $\mathrm{U}$ satisfying the inequality

$$
\|\mathcal{T} x\|_{Y} \leqslant \mathrm{U}\|x\|_{X}
$$

where $\|\cdot\|_{X}$ and $\|\cdot\|_{Y}$ are the norms on the spaces $X$ and $Y$, respectively. Here, $U$ does not depend on $X$. The best possible value of $U$ is regarded as the operator norm of $\mathcal{T}$.

The Euler mean matrix $\mathbf{E}^{r}$ of order $r$ is defined by the matrix $\mathbf{E}^{r}=\left(e_{n k}^{r}\right)$, where $0<r<1$ and

$$
e_{n k}^{r}= \begin{cases}\left(\begin{array}{l}
n \\
k
\end{array}\right)(1-r)^{n-k} r^{k}, & (0 \leqslant k \leqslant n), \\
0, & (k>n) .\end{cases}
$$

The Euler sequence spaces $\mathbf{e}_{\mathrm{p}}^{r}$ and $\mathbf{e}_{\infty}^{r}$ were introduced by Altay et al. [7] as follows (also see Altay and Başar [6]):

$$
\mathbf{e}_{p}^{r}=\left\{\mathbf{x}=\left(x_{k}\right) \in \omega: \sum_{n=0}^{\infty}\left|\sum_{k=0}^{n}\left(\begin{array}{l}
n \\
k
\end{array}\right)(1-r)^{n-k} r^{k} x_{k}\right|^{p}<\infty\right\}
$$

and

$$
\mathbf{e}_{\infty}^{r}=\left\{\mathbf{x}=\left(x_{k}\right) \in \omega: \sup _{n \in \mathbb{N}_{0}}\left|\sum_{k=0}^{n}\left(\begin{array}{l}
n \\
k
\end{array}\right)(1-r)^{n-k} r^{k} x_{k}\right|<\infty\right\} .
$$

Let $r, s \in \mathbb{R}$ and $r+s \neq 0$, then the binomial matrix $\mathbf{B}^{r, s}=\left(b_{n k}^{r, s}\right)$ is defined by:

$$
b_{n k}^{r, s}= \begin{cases}\frac{1}{(s+r)^{n}}\left(\begin{array}{l}
n \\
k
\end{array}\right) s^{n-k} r^{k}, & (0 \leqslant k \leqslant n), \\
0, & (k>n) .\end{cases}
$$


Bişgin $[10,11]$ obtained another generalization of Euler sequence spaces by introducing the binomial sequence spaces $\mathbf{b}_{\mathrm{p}}^{r, s}$ and $\mathbf{b}_{\infty}^{r, s}$ as follows:

$$
\mathbf{b}_{p}^{r, s}=\left\{\mathbf{x}=\left(x_{k}\right) \in \omega: \sum_{n=0}^{\infty}\left|\frac{1}{(s+r)^{n}} \sum_{k=0}^{n}\left(\begin{array}{l}
n \\
k
\end{array}\right) s^{n-k} r^{k} x_{k}\right|^{p}<\infty\right\}
$$

and

$$
\mathbf{b}_{\infty}^{r, s}=\left\{\mathbf{x}=\left(x_{k}\right) \in \omega: \sup _{n \in \mathbb{N}_{0}}\left|\frac{1}{(s+r)^{n}} \sum_{k=0}^{n}\left(\begin{array}{l}
n \\
k
\end{array}\right) s^{n-k} r^{k} x_{k}\right|<\infty\right\} .
$$

It is clear that, if we take $r+s=1$, then the binomial matrix $\mathbf{B}^{r, s}$ reduces to the Euler mean matrix $\mathbf{E}^{\mathrm{r}}$ of order $r$. Thus binomial matrix generalizes the Euler mean matrix.

Euler weighted sequence space $\mathbf{e}_{w, p}^{\theta}$ has been studied recently by Talebi and Dehgan [37] as follows:

$$
\mathbf{e}_{w, p}^{\theta}=\left\{\mathbf{x}=\left(x_{k}\right) \in \omega: \sum_{n=0}^{\infty} w_{n}\left|\sum_{k=0}^{n}\left(\begin{array}{l}
n \\
k
\end{array}\right)(1-\theta)^{n-k} \theta^{k} x_{k}\right|^{p}<\infty\right\},
$$

where $1 \leqslant p<\infty, 0<\theta<1$ and $w=\left(w_{n}\right)$ is a decreasing non-negative sequences of real numbers with $\sum_{n=0}^{\infty} \frac{w_{n}}{n+1}=\infty$

More recently, Manna [26] has studied Orlicz extension of weighted Euler sequence space and obtained norm inequalities involving generalized Hausdorff and Nörlund matrix operators which strengthen the results of Talebi and Dehgan [38]. The lower bounds of operators on different sequence spaces were studied in [18, 21-24]. Recently Roopaei and Foroutannia [34, 35] and Ilkhan [15] discussed the norms of matrix operators on different sequence spaces. Following Bişgin [10], Manna [26], Talebi and Dehgan [37], we introduce Orlicz extension of binomial sequence spaces $\mathbf{b}_{\varphi}^{r, s}$.

The paper is organized as follows. In the Section 2, we introduce Orlicz-binomial sequence space $\mathbf{b}_{\varphi}^{r, s}$, investigate topological properties and inclusion relations. In the Section 3, we give an upper bound estimation for the norm of Hausdorff matrix as an operator from $\ell_{\varphi}$ to $\mathbf{b}_{\varphi}^{r, s}$ and provide some immediate corollaries. In the Section 4, we give an upper bound estimation for $\|\mathbf{N}\|_{\ell_{\varphi}, b_{\varphi}^{r, s}}$ where $\mathbf{N}=\mathbf{N}\left(x_{n}\right)$ is the Nörlund matrix associated with the sequence $\mathbf{x}=\left(x_{n}\right)$. In the final section, we introduce operator ideal $\mathcal{L}_{\mathbf{b}_{\varphi}^{r, s}}^{(s)}$ using the space $\mathbf{b}_{\varphi}^{r, s}$ and the sequence of s-number functions and prove its completeness under certain assumption.

\section{Orlicz-binomial sequence spaces $b_{\varphi}^{r, s}$}

Let $\varphi$ be an Orlicz function. Then the Orlicz-binomial sequence space $\mathbf{b}_{\varphi}^{r, s}$ can be defined as the set of all sequences whose $\mathbf{B}^{r, s}$-transform is in $\ell_{\varphi}$. That is

$$
\mathbf{b}_{\varphi}^{r, s}=\left\{\mathbf{x} \in \omega: \mathbf{B}^{r, s} \mathbf{x} \in \ell_{\varphi}\right\}=\left\{\mathbf{x} \in \omega: \sum_{n=0}^{\infty} \varphi\left(\frac{1}{\rho} \sum_{k=0}^{n} \frac{1}{(s+r)^{n}}\left(\begin{array}{l}
n \\
k
\end{array}\right) s^{n-k_{r} k} x_{k}\right)<\infty \text { for some } \rho>0\right\} .
$$

One can observe that the sequence space $\mathbf{b}_{\varphi}^{r, s}$ reduces to $\mathbf{b}_{p}^{r, s}$ when $\varphi(t)=t^{p}, p \geqslant 1$ as studied by Bişgin [11] which further reduces to the space $\mathbf{b}_{p}^{r}$ as studied by Altay et al. [7] when $r+s=1$. Also if $r+s=1$, the sequence space $\mathbf{b}_{\varphi}^{r, s}$ reduces to the Orlicz-Euler sequence space $\mathbf{e}_{\varphi}^{r}$ studied by Manna [26].

Clearly the space $\mathbf{b}_{\varphi}^{r, s}$ is a normed linear space equipped with the norm $\|\mathbf{x}\|_{\varphi}^{r, s}=\left\|\mathbf{B}^{r, s} \mathbf{x}\right\|_{\varphi}$. We begin with the following theorem.

Theorem 2.1. The sequence space $\boldsymbol{b}_{\varphi}^{\mathrm{r}, \mathrm{s}}$ is a Banach space equipped with the norm $\|\cdot\|_{\varphi}^{\mathrm{r}, \mathrm{s}}$. 
Proof. Let $\left(x^{i}\right)$ be a Cauchy sequence in $\mathbf{b}_{\varphi}^{r, s}$. Then for any $\varepsilon>0$, there exist $n_{0} \in \mathbb{N}_{0}$ such that

$$
\left\|x^{i}-x^{j}\right\|_{\varphi}^{r, s}<\varepsilon \text { for each } i, j \geqslant n_{0}
$$

Choose $)<\rho_{\varepsilon}<\varepsilon$ such that

$$
\sum_{n=0}^{\infty} \varphi\left(\frac{1}{\rho_{\varepsilon}} \sum_{k=0}^{n} \frac{1}{(s+r)^{n}}\left(\begin{array}{l}
n \\
k
\end{array}\right) s^{n-k} r^{k}\left(x_{k}^{i}-x_{k}^{j}\right)\right) \leqslant 1 \text { holds for each } i, j \geqslant n_{0} .
$$

Using the assumption $\varphi(1)=1$, we obtain

$$
\frac{1}{\rho_{\varepsilon}} \sum_{k=0}^{n} \frac{1}{(s+r)^{n}}\left(\begin{array}{l}
n \\
k
\end{array}\right) s^{n-k} r^{k}\left(x_{k}^{i}-x_{k}^{j}\right) \leqslant 1 \text { holds for each } i, j \geqslant n_{0} .
$$

Thus it is clear that the sequence $\left(x_{k}^{i}\right)$ is a Cauchy sequence of real numbers and hence converges. Let $\left(x_{k}^{i}\right) \rightarrow x_{k}$ as $i \rightarrow \infty$ for each $k \geqslant 0$. Since $\varphi$ is continuous, we obtain from (2.1)

$$
\sum_{n=0}^{\infty} \varphi\left(\frac{1}{\rho_{\varepsilon}} \sum_{k=0}^{n} \frac{1}{(s+r)^{n}}\left(\begin{array}{l}
n \\
k
\end{array}\right) s^{n-k} r^{k}\left(x_{k}^{i}-x_{k}\right)\right) \leqslant 1 \text { holds for each } i \geqslant n_{0} .
$$

Thus $\mathbf{x} \in \mathbf{b}_{\varphi}^{r, s}$ and $\left\|x^{i}-x\right\|_{\varphi} \leqslant \rho_{\varepsilon}<\varepsilon$ for $i \geqslant n_{0}$. So $\left(\mathbf{b}_{\varphi}^{r, s},\|\cdot\|_{\varphi}^{r, s}\right)$ is a Banach space.

Theorem 2.2. The sequence space $\boldsymbol{b}_{\varphi}^{\mathrm{r}, \mathrm{s}}$ is linearly isomorphic to $\ell_{\varphi}$.

Proof. Define a mapping $\mathcal{T}: \mathbf{b}_{\varphi}^{r, s} \rightarrow \ell_{\varphi}$ by $\mathbf{x} \mapsto \mathbf{y}=\mathcal{T} \mathbf{x}$, where the sequence $\mathbf{y}=\left(\mathrm{y}_{\mathrm{k}}\right)$ is the $\mathbf{B}^{\mathrm{r}, \mathrm{s}}$-transform of the sequence $\mathbf{x}=\left(x_{k}\right)$, i.e.,

$$
y_{k}=\sum_{j=0}^{k} \frac{1}{(s+r)^{k}}\left(\begin{array}{l}
k \\
j
\end{array}\right) s^{k-j} r^{j} x_{j}
$$

Clearly, $\mathcal{T}$ is linear and injective. Let $\mathbf{y} \in \ell_{\varphi}$ and define the sequence $\mathbf{x}=\left(x_{k}\right)$ by

$$
x_{k}=\sum_{i=0}^{k}(s+r)^{i}\left(\begin{array}{l}
k \\
i
\end{array}\right)(-s)^{k-j} r^{-k} y_{i}
$$

Then, one obtains

$$
\begin{aligned}
\|\mathbf{x}\|_{\varphi}^{r, s}=\left\|\mathbf{B}^{r, s} \mathbf{x}\right\|_{\varphi} & =\sum_{k=0}^{\infty} \varphi\left(\frac{\mathbf{B}^{r, s} \mathbf{x}}{\rho}\right) \\
& =\sum_{k=0}^{\infty} \varphi\left(\frac{1}{\rho} \sum_{j=0}^{k} \frac{1}{(s+r)^{k}}\left(\begin{array}{l}
k \\
j
\end{array}\right) s^{k-j} r^{j} x_{j}\right) \\
& =\sum_{k=0}^{\infty} \varphi\left(\frac{1}{\rho} \sum_{j=0}^{k} \frac{1}{(s+r)^{k}}\left(\begin{array}{l}
k \\
j
\end{array}\right) s^{k-j} r^{j} \sum_{i=0}^{j}(s+r)^{i}\left(\begin{array}{l}
j \\
i
\end{array}\right)(-s)^{j-i} r^{-j} y_{i}\right) \\
& =\sum_{k=0}^{\infty} \varphi\left(\frac{\sum_{j=0}^{k} \delta_{k j} y_{j}}{\rho}\right)=\sum_{k=0}^{\infty} \varphi\left(\frac{y_{k}}{\rho}\right)=\|\mathbf{y}\|_{\varphi} \leqslant \rho .
\end{aligned}
$$

Thus we conclude that $\mathbf{x} \in \mathbf{b}_{\varphi}^{r, s}$ and $\mathcal{T}$ is norm preserving. Consequently, $\mathcal{T}$ is surjective. Thus $\mathbf{b}_{\varphi}^{r, s} \cong$ $\ell_{\varphi}$. 
Now we establish certain inclusion properties concerning Orlicz-binomial sequence space. We start with the following result.

Theorem 2.3. Let $\varphi$ be an Orlicz and supermultiplicative function. Then the inclusion $\ell_{\varphi} \subset \boldsymbol{b}_{\varphi}^{\mathrm{r}, \mathrm{s}}$ holds.

Proof. Let $\mathbf{x}=\left(x_{k}\right) \in \ell_{\varphi}$ with $x \neq 0$. Applying Jensen's inequality, we have

$$
\begin{aligned}
\sum_{n=0}^{\infty} \varphi\left(\frac{\mathbf{B}^{r, s} \mathbf{x}}{\rho}\right) & \leqslant \sum_{n=0}^{\infty} \sum_{k=0}^{n} \frac{1}{(s+r)^{n}}\left(\begin{array}{l}
n \\
k
\end{array}\right) s^{n-k} r^{k} \varphi\left(\frac{x_{k}}{\rho}\right) \\
& =\sum_{k=0}^{\infty} \varphi\left(\frac{x_{k}}{\rho}\right) \sum_{n=k}^{\infty}\left(\begin{array}{l}
n \\
k
\end{array}\right)\left(\frac{s}{s+r}\right)^{n-k}\left(\frac{r}{s+r}\right)^{k} \\
& =\frac{s+r}{r} \sum_{k=0}^{\infty} \varphi\left(\frac{x_{k}}{\rho}\right) \\
& =\sum_{k=0}^{\infty} \varphi\left(\frac{x_{k}}{\rho}\right) \varphi\left(\varphi^{-1}\left(\frac{s+r}{r}\right)\right) \leqslant \sum_{k=0}^{\infty} \varphi\left(\frac{x_{k}}{\rho} \varphi^{-1}\left(\frac{s+r}{r}\right)\right) .
\end{aligned}
$$

Let us put $\rho=\|\mathbf{x}\|_{\varphi} \varphi^{-1}\left(\frac{s+r}{r}\right)$. Then the above inequality implies that

$$
\sum_{n=0}^{\infty} \varphi\left(\frac{\mathbf{B}^{r, s} \mathbf{x}}{\rho}\right) \leqslant \sum_{k=0}^{\infty} \varphi\left(\frac{x_{k}}{\rho} \varphi^{-1}\left(\frac{s+r}{r}\right)\right)=\sum_{k=0}^{\infty} \varphi\left(\frac{x_{k}}{\|\mathbf{x}\|_{\varphi}}\right) \leqslant 1 .
$$

Hence, by the definition of Orlicz-Luxemborg norm, we obtain that

$$
\|\mathbf{x}\|_{\varphi}^{r, s} \leqslant \rho=\varphi^{-1}\left(\frac{s+r}{r}\right)\|\mathbf{x}\|_{\varphi} .
$$

Therefore, $\ell_{\varphi} \subset \mathbf{b}_{\varphi}^{r, s}$. To establish the strictness part, we consider $\varphi(t)=t^{p}, p \geqslant 1$. Then the sequence $\mathbf{x}=\left(x_{k}\right)=(-1)^{k} \in \mathbf{b}_{\varphi}^{r, s}$ but $\mathbf{x} \notin \ell_{\varphi}$. This completes the proof.

Theorem 2.4. Let $\varphi$ be an Orlicz and supermultiplicative function. Then the inclusion $\boldsymbol{e}_{\varphi}^{\mathrm{r}} \subset \boldsymbol{b}_{\varphi}^{\mathrm{r}, \mathrm{s}}$ is strict.

Proof. The inclusion part is obvious since the sequence space $\mathbf{b}_{\varphi}^{r, s}$ reduces to $\mathbf{e}_{\varphi}^{r}$ when $r+s=1$. To establish the strictness part, we consider $\varphi(t)=t^{p}, p \geqslant 1$ and a sequence $x=\left(x_{k}\right)=\left(\frac{-2}{r}\right)^{k}$ and let $0<r<1$ and $s=4$. Then one can easily deduce that $\left(x_{k}\right)=\left(\frac{-2}{r}\right)^{k} \notin \ell_{\varphi}, \mathbf{E}^{r}(x)=(-2-r)^{k} \notin \ell_{\varphi}$ and $\mathbf{B}^{r, s} \chi=\left(\frac{1}{s+r}\right)^{k} \in \ell_{\varphi}$. Thus there exists at least one sequence $\mathbf{x}=\left(x_{k}\right) \in \mathbf{b}_{\varphi}^{r, s} \backslash \mathbf{e}_{\varphi}^{r}$. This establishes the result.

\section{Upper bound for Hausdorff matrix operators}

In this section, we establish a Hardy type formula as an upper estimate for $\left\|\mathbf{H}_{\mu}\right\|_{\ell_{\varphi}, b_{\varphi}^{r, s}}$, where $d \mu$ is a Borel probability measure on $[0,1]$ and $\mathbf{H}_{\mu}$ is the generalized Hausdorff matrix associated with $d \mu$. Let $a>-1$ and $c>0$, then the generalized Hausdorff matrix, $\mathbf{H}_{\mu}=\mathbf{H}_{\mu}(\theta)=\left(h_{n k}(\theta)\right)_{n, k \geqslant 0}$ is defined by (see $[9,17])$

$$
h_{n k}= \begin{cases}\left(\begin{array}{l}
n+a \\
n-k
\end{array}\right) \Delta^{n-k} \mu_{k}, & (k \leqslant n), \\
0, & (k>n) .\end{cases}
$$

where $\Delta \mu_{k}=\mu_{k}-\mu_{k+1}$ and $\mu=\left(\mu_{n}\right)_{n \in \mathbb{N}}$ is a sequence of real numbers, normalized so that $\mu_{0}=1$ and

$$
\mu_{k}=\int_{0}^{1} \theta^{k} \mathrm{~d} \mu(\theta), \quad k=0,1,2, \ldots
$$


An equivalent expression for the generalized Hausdorff matrix $\mathbf{H}_{\mu}=\left(h_{n k}\right)$ is given by

$$
h_{n k}= \begin{cases}\left(\begin{array}{l}
n+a \\
n-k
\end{array}\right) \int_{0}^{1} \theta^{c(k+a)}\left(1-\theta^{c}\right)^{n-k} d \mu(\theta), & (k \leqslant n), \\
0, & (k>n) .\end{cases}
$$

When $a=0$ and $c=1$, then $\mathbf{H}_{\mu}$ reduces to the ordinary Hausdorff matrix (see [9]) which generalizes various classes of matrices. These classes are:

(a) taking $\mathrm{d} \mu(\theta)=\alpha(1-\theta)^{\alpha-1} \mathrm{~d} \theta$ gives the Cesàro matix of order $\alpha$;

(b) taking $d \mu(\theta)=$ point evaluation at $\theta=\alpha$ gives the Euler matrix of order $\alpha$;

(c) taking $\mathrm{d} \mu(\theta)=|\log \theta|^{\alpha-1} / \Gamma(\alpha) \mathrm{d} \theta$ gives the Hölder matrix of order $\alpha$;

(d) taking $\mathrm{d} \mu(\theta)=\alpha \theta^{\alpha-1} \mathrm{~d} \theta$ gives the Gamma matrix of order $\alpha$.

The Cesàro, Hölder, and Gamma matrices have non negative entries when $\alpha>0$, and also the Euler matrices, when $0<\alpha<1$. Now we consider the following hypothesis related to Orlicz function and Hausdorff matrix:

'Hypothesis $\mathbf{O H}^{\prime}$ : Let $\varphi$ be an Orlicz and supermultiplicative function, $\varphi^{-1}$ be its inverse, and $\|\cdot\|_{\varphi}$ be the Orlicz-Luxemborg norm. Denote $(x)_{q}=\frac{\Gamma(x+q)}{x}$ for $x \geqslant 0$ and $H_{\mu}=\left(h_{n k}\right), h_{n k} \geqslant 0$. Further, let $a>-1, c>0, q>-a-1$ and $\frac{1}{(n+a+1)_{q}}$ be non-increasing for $n \in \mathbb{N}_{0}$.

Now we state a lemma due to Love [25] which is essential for deducing our results.

Lemma 3.1 ([25, Theorem 2]). Suppose that the 'Hypothesis $\mathbf{O H}^{\prime}$ holds. Then for any non-negative sequence $x=\left(x_{k}\right)$ and $\mu=\left(\mu_{k}\right)$ of real numbers normalized so that $\mu_{0}=1$, the following inequality holds:

$$
\left\|\boldsymbol{H}_{\mu} x\right\|_{\varphi} \leqslant \tilde{\mathrm{C}}\|\boldsymbol{x}\|_{\varphi},
$$

where

$$
\tilde{C}=\int_{0}^{1} \varphi^{-1}\left(\theta^{-(\mathbf{q}+1) \mathbf{c}}\right) d \mu(\theta) .
$$

Theorem 3.2. Suppose that the 'Hypothesis $\mathbf{O H}^{\prime}$ holds. Then the Hausdorff matrix $\boldsymbol{H}_{\mu}$ maps $\ell_{\varphi}$ to $\boldsymbol{b}_{\varphi}^{\mathrm{r}, \mathrm{s}}$ and

$$
\left\|\boldsymbol{H}_{\mu}\right\|_{\ell_{\varphi}, b_{\varphi}^{r, s}} \leqslant \tilde{\mathrm{C}} \varphi^{-1}\left(\frac{s+r}{r}\right)
$$

where $\tilde{\mathrm{C}}$ is given by (3.2).

Proof. Let $\mathbf{x}=\left(x_{n}\right)$ be a non negative sequence of real numbers in $\ell_{\varphi}$. Let $\rho>0$ be a real number, then using Jensen's inequality, we have

$$
\begin{aligned}
\left\|\mathbf{H}_{\mu}\right\|_{\ell_{\varphi}, b_{\varphi}^{r, s}} & =\sum_{n=0}^{\infty} \varphi\left(\frac{1}{\rho} \sum_{k=0}^{n} \frac{1}{(s+r)^{n}}\left(\begin{array}{l}
n \\
k
\end{array}\right) s^{n-k^{k}} \sum_{i=0}^{k} h_{k i} x_{i}\right) \\
& \leqslant \sum_{n=0}^{\infty} \sum_{k=0}^{n} \frac{1}{(s+r)^{n}}\left(\begin{array}{l}
n \\
k
\end{array}\right) s^{n-k} r^{k} \varphi\left(\frac{1}{\rho} \sum_{i=0}^{k} h_{k i} x_{i}\right) \\
& \leqslant \sum_{k=0}^{\infty} \varphi\left(\frac{1}{\rho} \sum_{i=0}^{k} h_{k i} x_{i}\right) \sum_{n=k}^{\infty}\left(\begin{array}{l}
n \\
k
\end{array}\right)\left(\frac{s}{s+r}\right)^{n-k}\left(\frac{r}{s+r}\right)^{k} \\
& =\frac{s+r}{r} \sum_{k=0}^{\infty} \varphi\left(\frac{1}{\rho} \sum_{i=0}^{k} h_{k i} x_{i}\right) \\
& =\sum_{k=0}^{\infty} \varphi\left(\frac{1}{\rho} \sum_{i=0}^{k} h_{k i} x_{i}\right) \varphi\left(\varphi^{-1}\left(\frac{s+r}{r}\right)\right)
\end{aligned}
$$




$$
\leqslant \sum_{k=0}^{\infty} \varphi\left(\frac{1}{\rho} \sum_{i=0}^{k} h_{k i} x_{i} \varphi^{-1}\left(\frac{s+r}{r}\right)\right)
$$

Let $\rho=\left\|\mathbf{H}_{\mu} x\right\|_{\varphi} \varphi^{-1}\left(\frac{s+r}{r}\right)$. Then the above inequality implies that

$$
\left\|\mathbf{H}_{\mu}\right\|_{\ell_{\varphi}, \mathrm{b}_{\varphi}^{\mathrm{r}, s}} \leqslant \sum_{\mathrm{k}=0}^{\infty} \varphi\left(\frac{1}{\rho} \sum_{i=0}^{\mathrm{k}} h_{\mathrm{ki}} x_{i} \varphi^{-1}\left(\frac{\mathrm{s}+\mathrm{r}}{\mathrm{r}}\right)\right)=\sum_{\mathrm{k}=0}^{\infty} \varphi\left(\frac{\mathbf{H}_{\mu} \mathbf{x}}{\left\|\mathbf{H}_{\mu} \mathbf{x}\right\|_{\varphi}}\right) \leqslant 1 .
$$

Now using the definition of Orlicz-Luxemborg norm and equation (3.1), we get

$$
\left\|\mathbf{H}_{\mu} \mathbf{x}\right\|_{\varphi}^{r, s} \leqslant \rho=\left\|\mathbf{H}_{\mu} \mathbf{x}\right\|_{\varphi} \varphi^{-1}\left(\frac{s+r}{r}\right) \leqslant \tilde{C} \varphi^{-1}\left(\frac{s+r}{r}\right)\|\mathbf{x}\|_{\varphi} .
$$

This gives

$$
\left\|\mathbf{H}_{\mu}\right\|_{\ell_{\varphi}, \mathbf{b}_{\varphi}^{r, s}} \leqslant \tilde{C} \varphi^{-1}\left(\frac{s+r}{r}\right)
$$

Corollary 3.3. Choose $\mathrm{c}=1$ and $\mathrm{a}=0$. Then $\tilde{\mathrm{C}}=\int_{0}^{1} \varphi^{-1}\left(\theta^{-(\mathrm{q}+1)}\right) \mathrm{d} \mu(\theta)$ and Cesàro, Hölder, Euler, and Gamma matrices map $\ell_{\varphi}$ into $\boldsymbol{b}_{\varphi}^{\mathrm{r}, \mathrm{s}}$ and

$$
\begin{aligned}
& \|\boldsymbol{C}(\alpha)\|_{\ell_{\varphi}, b_{\varphi}^{r, s}} \leqslant \alpha \varphi^{-1}\left(\frac{s+r}{r}\right) \int_{0}^{1} \varphi^{-1}\left(\theta^{-(q+1)}\right)(1-\theta)^{\alpha-1} \mathrm{~d} \theta, \alpha>0 ; \\
& \|\boldsymbol{H}(\alpha)\|_{\ell_{\varphi}, b_{\varphi}^{r, s}} \leqslant \frac{1}{\Gamma(\alpha)} \varphi^{-1}\left(\frac{s+r}{r}\right) \int_{0}^{1} \varphi^{-1}\left(\theta^{-(q+1)}\right)|\log \theta|^{\alpha-1} \mathrm{~d} \theta, \alpha>0 ; \\
& \|\boldsymbol{E}(\alpha)\|_{\ell_{\varphi}, b_{\varphi}^{r, s}} \leqslant \varphi^{-1}\left(\frac{s+r}{r}\right) \varphi^{-1}\left(\alpha^{-(q+1)}\right), 0<\alpha<1 ; \\
& \|\Gamma(\alpha)\|_{\ell_{\varphi}, b_{\varphi}^{r, s}} \leqslant \alpha \varphi^{-1}\left(\frac{s+r}{r}\right) \int_{0}^{1} \varphi^{-1}\left(\theta^{-(q+1)}\right) \theta^{\alpha-1} d \theta .
\end{aligned}
$$

Corollary 3.4. Choose $\mathrm{c}=1, \mathrm{a}=0, \mathrm{q}=0$ and $\varphi(\mathrm{t})=\mathrm{t}^{\mathrm{p}}, \mathrm{p} \geqslant 1$, and denote $\mathrm{p}^{*}=\frac{\mathrm{p}}{\mathrm{p}-1}$. Then Cesàro, Hölder, Euler and Gamma matrices map $\ell_{\varphi}$ to $\boldsymbol{b}_{\varphi}^{\mathrm{r}, \mathrm{s}}$ and

$$
\begin{aligned}
& \|\boldsymbol{C}(\alpha)\|_{\ell_{\varphi}, b_{\varphi}^{r, s}} \leqslant\left(\frac{s+r}{r}\right)^{\frac{1}{p}} \frac{\Gamma(\alpha+1) \Gamma\left(\frac{1}{p^{*}}\right)}{\Gamma\left(\alpha+\frac{1}{p^{*}}\right)}, \quad \alpha>0 ; \\
& \|H(\alpha)\|_{\ell_{\varphi}, b_{\varphi}^{r, s}} \leqslant\left(\frac{s+r}{r}\right)^{\frac{1}{p}} \frac{1}{\Gamma(\alpha)} \int_{0}^{1} \theta^{\frac{-1}{p}}|\log \theta|^{\alpha-1} d \theta, \quad \alpha>0 ; \\
& \|E(\alpha)\|_{\ell_{\varphi}, b_{\varphi}^{r, s}} \leqslant\left(\frac{s+r}{r}\right)^{\frac{1}{p}} \alpha^{\frac{-1}{p}}, \quad 0<\alpha<1 ; \\
& \|\Gamma(\alpha)\|_{\ell_{\varphi}, b_{\varphi}^{r, s}} \leqslant\left(\frac{s+r}{r}\right)^{\frac{1}{p}} \frac{\alpha p}{\alpha p-1}, \quad \alpha p>1 .
\end{aligned}
$$

\section{Upper bound for Nörlund matrix operator}

In this section, we give an upper bound estimation for the norm of Nörlund matrix as an operator 
from $\ell_{\varphi}$ to $\mathbf{b}_{\varphi}^{r, s}$. Let $u=\left(u_{n}\right)$ be a sequence of non-negative numbers with $u_{0}>0$. We write $u_{n}=$ $\sum_{k=0}^{n} u_{k}, n \geqslant 0$. Then the Nörlund mean with respect to the sequence $u=\left(u_{n}\right)$ is defined by the matrix $\mathbf{N}=\mathbf{N}\left(u_{n}\right)=\left(a_{n k}^{u}\right)$ given by

$$
a_{n k}^{u}= \begin{cases}\frac{u_{n-k}}{u_{n}}, & (0 \leqslant k \leqslant n) \\ 0, & k>n .\end{cases}
$$

In the case when $u_{n}=e$, Nörlund matrix reduces to Cesàro matrix. Note that one can assume $u_{0}=1$ because $\mathbf{N}\left(u_{n}\right)=\mathbf{N}\left(c u_{n}\right)$ for any $c>0$.

Theorem 4.1. Let $u=\left(u_{n}\right)$ be a sequence of non-negative real numbers with $u_{0}=1$. Then

$$
\|N\|_{\ell_{\varphi}, b_{\varphi}^{r, s}} \leqslant \varphi^{-1}\left(\frac{s+r}{r} \sum_{n=0}^{\infty} \frac{u_{n}}{u_{n}}\right) .
$$

Proof. Let $x \in \ell_{\varphi}$ be any non-negative sequence of real numbers and $\rho>0$. Applying Jensen's inequality, we obtain

$$
\begin{aligned}
\sum_{n=0}^{\infty} \varphi\left(\frac{1}{\rho} \sum_{k=0}^{n} \frac{1}{(s+r)^{n}}\left(\begin{array}{l}
n \\
k
\end{array}\right) s^{n-k} r^{k} \sum_{i=0}^{k} \frac{u_{k-i}}{u_{k}} x_{i}\right) & \leqslant \sum_{n=0}^{\infty} \sum_{k=0}^{n} \frac{1}{(s+r)^{n}}\left(\begin{array}{l}
n \\
k
\end{array}\right) s^{n-k} r^{k} \varphi\left(\frac{1}{\rho} \sum_{i=0}^{k} \frac{u_{k-i}}{u_{k}} x_{i}\right) \\
& =\sum_{k=0}^{\infty} \varphi\left(\frac{1}{\rho} \sum_{i=0}^{k} \frac{u_{k-i}}{u_{k}} x_{i}\right) \sum_{n=k}^{\infty}\left(\begin{array}{l}
n \\
k
\end{array}\right)\left(\frac{s}{s+r}\right)^{n-k}\left(\frac{r}{s+r}\right)^{k} \\
& \leqslant \frac{s+r}{r} \sum_{k=0}^{\infty} \sum_{i=0}^{k} \frac{u_{k-i}}{u_{k}} \varphi\left(\frac{x_{i}}{\rho}\right) \\
& =\frac{s+r}{r} \sum_{i=0}^{\infty} \varphi\left(\frac{x_{i}}{\rho}\right) \sum_{k=i}^{\infty} \frac{u_{k-i}}{u_{k}} \\
& \leqslant \frac{s+r}{r} \sum_{k=0}^{\infty} \frac{u_{k}}{u_{k}} \sum_{i=0}^{\infty} \varphi\left(\frac{x_{i}}{\rho}\right) \\
& =\sum_{i=0}^{\infty} \varphi\left(\frac{x_{i}}{\rho}\right) \varphi\left(\varphi-1\left(\frac{s+r}{r} \sum_{k=0}^{\infty} \frac{u_{k}}{u_{k}}\right)\right) \\
& \leqslant \sum_{i=0}^{\infty} \varphi\left(\frac{x_{i}}{\rho} \varphi^{-1}\left(\frac{s+r}{r} \sum_{k=0}^{\infty} \frac{u_{k}}{u_{k}}\right)\right)
\end{aligned}
$$

Put $\rho=\|x\|_{\ell_{\varphi}} \varphi^{-1}\left(\frac{s+r}{r} \sum_{k=0}^{\infty} \frac{\mathfrak{u}_{k}}{u_{k}}\right)$, then the above inequality becomes

$$
\|\mathbf{N} \mathbf{x}\|_{\ell_{\varphi}, \mathbf{b}_{\varphi}^{r, s}} \leqslant \sum_{i=0}^{\infty} \varphi\left(\frac{x_{i}}{\|x\|_{\ell_{\varphi}}}\right) \leqslant 1 .
$$

Now using the definition of Orlicz-Luxemborg norm, we get

$$
\|\mathbf{N} \boldsymbol{x}\|_{\ell_{\varphi}, \mathbf{b}_{\varphi}^{r, s}} \leqslant \rho=\varphi^{-1}\left(\frac{s+r}{r} \sum_{k=0}^{\infty} \frac{u_{k}}{u_{k}}\right)\|\mathbf{x}\|_{\ell_{\varphi}} .
$$

This establishes the result. 
Corollary 4.2. Let $\mathrm{u}=\left(\mathrm{u}_{\mathrm{n}}\right)$ be a non-negative sequence of real numbers such that $\frac{\mathrm{u}_{\mathrm{n}}}{\mathrm{u}_{\mathrm{n}}}=\frac{1}{(\mathrm{n}+1)^{2}}, \mathrm{n}=0,1,2, \ldots$ Then the Nörlund matrix maps $\ell_{\varphi}$ into $\boldsymbol{b}_{\varphi}^{\mathrm{r}, \mathrm{s}}$ and

$$
\|N\|_{\ell_{\varphi}, b_{\varphi}^{r, s}} \leqslant \varphi^{-1}\left(\frac{(s+r) \pi^{2}}{6 r}\right) .
$$

Corollary 4.3. Let $\varphi(t)=t^{p} p \geqslant 1$ and $u=\left(u_{n}\right)$ be a non-negative sequence of real numbers such that $\frac{\mathfrak{u}_{\mathrm{n}}}{\mathrm{u}_{\mathrm{n}}}=\frac{1}{(\mathrm{n}+1)^{2}}, \mathrm{n}=0,1,2, \ldots$ Then the Nörlund matrix maps $\ell_{\varphi}$ into $\boldsymbol{b}_{\varphi}^{\mathrm{r}, \mathrm{s}}$ and

$$
\|N\|_{\ell_{\varphi}, b_{\varphi}^{r, s}} \leqslant\left(\frac{(s+r) \pi^{2}}{6 r}\right)^{\frac{1}{p}} .
$$

\section{The operator ideals $\mathcal{L}_{\mathbf{b}_{\varphi}^{\text {r,s }}}^{(s)}$}

Throughout this section, we denote by $X$ and $Y$, the Banach spaces over the complex field $\mathbb{C}$ and by $\mathbf{L}(X, Y)$, the class of all bounded linear maps from $X$ to $Y$. Let $\mathbf{L}$ be the class of all bounded linear operators between any pair of Banach spaces.

A map s : $\mathbf{L} \rightarrow \omega^{+}$, where $\omega^{+}$is the class of sequences of non-negative real numbers, is called an s-number function if it satisfies the following conditions:

(i) $\|s\|=s_{0}(\mathcal{S}) \geqslant s_{1}(\mathcal{S}) \geqslant \cdots \geqslant 0, s(\mathcal{S})=\left\{s_{\mathfrak{n}}(\mathcal{S})\right\}, \mathcal{S} \in \mathbf{L}$;

(ii) $s_{n}(\mathcal{S}+\mathcal{T}) \leqslant s_{n}(\mathcal{S})+\|\mathcal{T}\|$ for $\mathcal{S}, \mathcal{T} \in \mathbf{L}(X, Y)$ and $n \in \mathbb{N}_{0}$;

(iii) $s_{n}(\mathcal{R S T}) \leqslant\|\mathcal{R}\| s_{n}(\mathcal{S})\|\mathcal{T}\|$ for $\mathcal{T} \in \mathbf{L}\left(X_{0}, X\right), \mathcal{S} \in \mathbf{L}(X, Y), \mathcal{R} \in \mathbf{L}\left(Y, Y_{0}\right)$, and $n \in \mathbb{N}_{0}$;

(iv) if $\operatorname{rank}(\mathcal{S})<\mathrm{n}$, then $s_{\mathfrak{n}}(\mathcal{S})=0$;

(v) if $\operatorname{dim} X \geqslant n$, then $s_{n}\left(\mathcal{J}_{X}\right)=1$, where $\mathcal{J}_{X}$ denotes the identity map of $X$.

An s-number function is called additive if the condition (ii) is replaced by

(ii) $s_{m+n-1}(\mathcal{S}+\mathcal{T}) \leqslant s_{m}(\mathcal{S})+s_{n}(\mathcal{T})$ for $\mathcal{S}, \mathcal{T} \in \mathbf{L}(X, Y)$ and $m, n \in \mathbb{N}_{0}$.

If the condition (iii) is replaced by

(iii) $s_{\mathrm{m}+\mathfrak{n}-1}(R T) \leqslant s_{m}(\mathcal{R}) s_{\mathfrak{n}}(\mathcal{T})$ for $\mathcal{R} \in \mathbf{L}\left(Y_{0}, Y\right)$ and $\mathcal{T} \in \mathbf{L}\left(X, Y_{0}\right), m, n \in \mathbb{N}_{0}$,

then the s-number function is called multiplicative, where the negative subscript is consider to be naught.

For a subset $\mathbf{A}$ of $\mathbf{L}$, we write $\mathbf{A}(X, Y)=\mathbf{A} \cap \mathbf{L}(X, Y)$ where $X$ and $Y$ are Banach spaces. The collection $\mathbf{A}$ is said to be an operator ideal if it satisfies the following conditions:

(i) A contains all finite rank operators;

(ii) $\mathcal{T}+\mathcal{S} \in \mathbf{A}(X, Y)$ for $\mathcal{S}, \mathcal{T} \in \mathbf{A}(X, Y)$;

(iii) if $\mathcal{T} \in \mathbf{A}(X, Y)$ and $\mathcal{S} \in \mathbf{L}(Y, Z)$, then $\mathcal{S} \mathcal{T} \in \mathbf{A}(X, Z)$ and also if $\mathcal{T} \in \mathbf{L}(X, Y)$ and $\mathcal{S} \in \mathbf{A}(Y, Z)$, then $\mathcal{S T} \in \mathbf{A}(X, Z)$.

The collection $\mathbf{A}(X, Y)$, for a given pair of Banach spaces $X$ and $Y$, is called a component of $\mathbf{A}$. For more details on s-number and operator ideal, we strictly refer to [1-4, 14, 19, 28-33] and the references cited therein.

An ideal quasi norm is a real valued function $f$ defined on an operator ideal $\mathbf{A}$, which satisfies the following properties:

(i) $0 \leqslant f(\mathcal{T})<\infty$, for each $\mathcal{T} \in \mathbf{A}$ and $f(\mathcal{T})=0$ if and only if $\mathcal{T}=0$;

(ii) there exists a constant $N \geqslant 1$ such that $f(\mathcal{S}+\mathcal{T}) \leqslant N[f(\mathcal{S})+f(\mathcal{T})]$ for $\mathcal{S}, \mathcal{T} \in \mathbf{A}(X, Y)$, where $\mathbf{A}(X, Y)$ is any component of $\mathbf{A}$;

(iii) (a) $f(\mathcal{R S}) \leqslant\|\mathcal{R}\| f(\mathcal{S})$ for $\mathcal{S} \in \mathbf{A}(X, Z), \mathcal{R} \in \mathbf{L}(Z, Y)$; and

(b) $f(\mathcal{R S}) \leqslant\|\mathcal{S}\| f(\mathcal{R})$ for $\mathcal{S} \in \mathbf{L}(X, Z), \mathcal{R} \in \mathbf{A}(Z, Y)$. 
We start with the following definition.

Definition 5.1. An operator $\mathcal{T} \in \mathbf{L}(X, Y)$ is said to be of type $\mathbf{b}_{\varphi}^{\mathrm{r}, \mathrm{s}}$ if $\left\{s_{\mathfrak{n}}(\mathcal{T})\right\} \in \mathbf{b}_{\varphi}^{\mathrm{r}, \mathrm{s}}$.

Let $\mathcal{L}_{\mathbf{b}_{\varphi}^{\text {r,s }}}^{(s)}$ denotes the collection of all such mappings, i.e.,

$$
\mathcal{L}_{\mathbf{b}_{\varphi}^{r, s}}^{(s)}=\left\{\mathcal{T} \in \mathbf{L}(X, Y):\left\{s_{n}(\mathcal{T})\right\} \in \mathbf{b}_{\varphi}^{r, s}\right\}
$$

For $\mathcal{T} \in \mathcal{L}_{\mathbf{b}_{\varphi}^{\text {r,s }}}^{(s)}$, we define

$$
\|\mathcal{T}\|_{\mathbf{b}_{\varphi}^{r, s}}^{(s)}=\inf \left\{\rho>0: \sum_{n=0}^{\infty} \varphi\left(\frac{1}{\rho} \sum_{k=0}^{n} \frac{1}{(s+r)^{n}}\left(\begin{array}{l}
n \\
k
\end{array}\right) s^{n-k_{r} k^{k}} s_{k}(\mathcal{T})\right) \leqslant 1\right\} .
$$

Theorem 5.2. The class $\mathcal{L}_{\boldsymbol{b}_{\varphi}^{\text {r.s }}}^{(\mathrm{s})}$ is an operator ideal equipped with the norm $\|\cdot\|_{\boldsymbol{b}_{\varphi}^{\mathrm{r}, \mathrm{s}}}^{(\mathrm{s})}$.

Proof. Note that all the finite rank operators are contained in $\mathcal{L}_{\mathbf{b}_{\varphi}^{r, s}}^{(s)}$, since $s_{n}(\mathcal{T})=0$ for $n \geqslant n_{0}$ if $\operatorname{rank}(\mathcal{T})<$ $\mathrm{n}_{0}$. Let $\mathcal{T}_{1}, \mathcal{T}_{2} \in \mathcal{L}_{\mathbf{b}_{\varphi}^{\text {r,s }}}^{(\mathrm{s})}$, then

$$
\begin{aligned}
& \sum_{n=0}^{\infty} \varphi\left(\frac{1}{\rho_{1}} \sum_{k=0}^{n} \frac{1}{(s+r)^{n}}\left(\begin{array}{l}
n \\
k
\end{array}\right) s^{n-k} r^{k} s_{k}\left(\mathcal{T}_{1}\right)\right)<\infty \text { for some } \rho_{1}>0, \text { and } \\
& \sum_{n=0}^{\infty} \varphi\left(\frac{1}{\rho_{2}} \sum_{k=0}^{n} \frac{1}{(s+r)^{n}}\left(\begin{array}{l}
n \\
k
\end{array}\right) s^{n-k} r^{k} s_{k}\left(\mathcal{T}_{2}\right)\right)<\infty \text { for some } \rho_{2}>0 .
\end{aligned}
$$

Now

$$
\begin{aligned}
& \sum_{n=0}^{\infty} \varphi\left(\frac{1}{\rho_{1}+\rho_{2}} \sum_{k=0}^{n} \frac{1}{(s+r)^{n}}\left(\begin{array}{l}
n \\
k
\end{array}\right) s^{n-k} r^{k} s_{k}\left(\mathcal{T}_{1}+\mathcal{T}_{2}\right)\right) \\
& \quad \leqslant \sum_{n=0}^{\infty} \sum_{k=0}^{n} \frac{1}{(s+r)^{n}}\left(\begin{array}{l}
n \\
k
\end{array}\right) s^{n-k^{k}} \varphi\left(\frac{1}{\rho_{1}+\rho_{2}} s_{k}\left(\mathcal{T}_{1}+\mathcal{T}_{2}\right)\right) \quad \text { (using Jensen's inequality) } \\
& \quad=\sum_{k=0}^{\infty} \varphi\left(\frac{1}{\rho_{1}+\rho_{2}} s_{k}\left(\mathcal{T}_{1}+\mathcal{T}_{2}\right)\right) \sum_{n=k}^{\infty} \frac{1}{(s+r)^{n}}\left(\begin{array}{l}
n \\
k
\end{array}\right) s^{n-k} r^{k} \\
& \quad=\frac{s+r}{r} \sum_{k=0}^{\infty} \varphi\left(\frac{1}{\rho_{1}+\rho_{2}} s_{k}\left(\mathcal{T}_{1}+\mathcal{T}_{2}\right)\right) \\
& \quad \leqslant \frac{s+r}{r}\left(\sum_{k=0}^{\infty} \frac{\rho_{1}}{\rho_{1}+\rho_{2}} \varphi\left(\frac{s_{k}\left(\mathcal{T}_{1}\right)}{\rho_{1}}\right)+\sum_{k=0}^{\infty} \frac{\rho_{1}}{\rho_{1}+\rho_{2}} \varphi\left(\frac{s_{k}\left(\mathcal{T}_{2}\right)}{\rho_{2}}\right)\right)<\infty .
\end{aligned}
$$

Thus $\mathcal{T}_{1}+\mathcal{T}_{2} \in \mathcal{L}_{\mathbf{b}_{\varphi}^{r, s}}^{(s)}$. Let $\mathcal{T} \in \mathcal{L}_{\mathbf{b}_{\varphi}^{r, s}}^{(s)}\left(X_{0}, Y_{0}\right), \mathcal{R} \in \mathcal{L}_{\mathbf{b}_{\varphi}^{r, s}}^{(s)}\left(X, X_{0}\right), \mathcal{S} \in \mathcal{L}_{\mathbf{b}_{\varphi}^{r, s}}^{(s)}\left(Y_{0}, Y\right)$. Using the property (iii) of s-number function, we obtain

$$
\begin{aligned}
& \sum_{n=0}^{\infty} \varphi\left(\frac{1}{\rho} \sum_{k=0}^{n} \frac{1}{(s+r)^{n}}\left(\begin{array}{l}
n \\
k
\end{array}\right) s^{n-k} r^{k} s_{k}(\mathcal{R T S})\right) \\
& \leqslant \sum_{n=0}^{\infty} \sum_{k=0}^{n} \frac{1}{(s+r)^{n}}\left(\begin{array}{l}
n \\
k
\end{array}\right) s^{n-k} r^{k} \varphi\left(\frac{1}{\rho} s_{k}(\mathcal{R T S})\right) \quad \text { (using Jensen's inequality) } \\
& \quad \leqslant \sum_{n=0}^{\infty} \sum_{k=0}^{n} \frac{1}{(s+r)^{n}}\left(\begin{array}{l}
n \\
k
\end{array}\right) s^{n-k} r^{k} \varphi\left(\frac{\|\mathcal{R}\|\|\mathcal{S}\|}{\rho} s_{k}(\mathcal{T})\right) \text { (using property (iii) of s-number function) }
\end{aligned}
$$




$$
=\frac{s+r}{r} \sum_{k=0}^{\infty} \varphi\left(\frac{\|\mathcal{R}\| s_{k}(\mathcal{T})\|\mathcal{S}\|}{\rho}\right)<\infty .
$$

Thus $\mathcal{R T S} \in \mathcal{L}_{\mathbf{b}_{\varphi}^{r, s}}^{(s)}$. Thus $\mathcal{L}_{\mathbf{b}_{\varphi}^{r, s}}^{(s)}$ is an operator ideal.

Theorem 5.3. The operator ideal $\mathcal{L}_{\boldsymbol{b}_{\varphi}^{\text {r.s }}}^{(\mathrm{s})}$ is complete under the quasi-norm $\|\cdot\|_{\boldsymbol{b}_{\varphi}^{\mathrm{r}, s}}^{(\mathrm{s})}$.

Proof. First we shall show that $\|\cdot\|_{\mathbf{b}_{\varphi}}^{\mathrm{r}, \mathrm{s}}$ is a quasi-norm on $\mathcal{L}_{\mathbf{b}_{\varphi}^{\mathrm{r}, s}}^{(s)}$. Note that $\|\mathcal{T}\|_{\mathbf{b}_{\varphi}^{r, s}}^{(s)} \geqslant 0$ for each $\mathcal{T} \in \mathcal{L}_{\mathbf{b}_{\varphi}^{r, s}}^{(s)}$ and $\|\mathcal{T}\|_{\mathbf{b}_{\varphi}^{r, s}}^{(s)}=0$ for $\mathcal{T}=0$. Now, let $\mathcal{T} \in \mathcal{L}_{\mathbf{b}_{\varphi}^{r, s}}^{(s)}$ such that $\|\mathcal{T}\|_{\mathbf{b}_{\varphi}^{r, s}}^{(s)}=0$. Then for $\varepsilon>0$, we can find $0<\rho<\varepsilon$ and

$$
\sum_{n=0}^{\infty} \varphi\left(\frac{1}{\rho} \sum_{k=0}^{n} \frac{1}{(s+r)^{n}}\left(\begin{array}{l}
n \\
k
\end{array}\right) s^{n-k} r^{k} s_{k}(\mathcal{T})\right) \leqslant 1 .
$$

Using the assumption $\varphi(1)=1$, one obtains

$$
\frac{1}{\varepsilon} \sum_{k=0}^{n} \frac{1}{(s+r)^{n}}\left(\begin{array}{l}
n \\
k
\end{array}\right) s^{n-k} r^{k} s_{k}(\mathcal{T}) \leqslant \frac{1}{\rho} \sum_{k=0}^{n} \frac{1}{(s+r)^{n}}\left(\begin{array}{l}
n \\
k
\end{array}\right) s^{n-k} r^{k} s_{k}(\mathcal{T}) \leqslant 1 .
$$

Now

$$
\frac{1}{\varepsilon}\left(\frac{s}{s+r}\right)^{n} s_{0}(\mathcal{T}) \leqslant \frac{1}{\varepsilon} \sum_{k=0}^{n} \frac{1}{(s+r)^{n}}\left(\begin{array}{l}
n \\
k
\end{array}\right) s^{n-k} r^{k} s_{k}(\mathcal{T}) \leqslant 1 .
$$

Since $\varepsilon$ is arbitrary, we get

$$
\|\mathcal{T}\|=\mathrm{s}_{0}(\mathcal{T})=0 \Longrightarrow \mathcal{T}=0 .
$$

Next we establish the triangular inequality. Let $\mathcal{T}_{1}, \mathcal{T}_{2} \in \mathcal{L}_{\mathbf{b}_{\varphi}^{\text {r,s }}}^{(s)}$ and $\varepsilon>0$ arbitrary. Choose $\rho_{1}>0, \rho_{2}>0$ such that

$$
\begin{aligned}
& \frac{1}{\rho_{1}} \sum_{k=0}^{n} \frac{1}{(s+r)^{n}}\left(\begin{array}{l}
n \\
k
\end{array}\right) s^{n-k} r^{k} s_{k}\left(\mathcal{T}_{1}\right) \leqslant 1, \quad \rho_{1} \leqslant\left\|\mathcal{T}_{1}\right\|_{\mathbf{b}_{\varphi}^{r, s}}^{(s)}+\frac{\varepsilon}{2}, \text { and } \\
& \frac{1}{\rho_{2}} \sum_{k=0}^{n} \frac{1}{(s+r)^{n}}\left(\begin{array}{l}
n \\
k
\end{array}\right) s^{n-k} r^{k} s_{k}\left(\mathcal{T}_{2}\right) \leqslant 1, \quad \rho_{2} \leqslant\left\|\mathcal{T}_{2}\right\|_{\mathbf{b}_{\varphi}^{r, s}}^{(s)}+\frac{\varepsilon}{2} .
\end{aligned}
$$

We choose $\mathrm{N}>1$. Then

$$
\sum_{n=0}^{\infty} \varphi\left(\frac{1}{N\left(\rho_{1}+\rho_{2}\right)} \sum_{k=0}^{n} \frac{1}{(s+r)^{n}}\left(\begin{array}{l}
n \\
k
\end{array}\right) s^{n-k} r^{k} s_{k}\left(\mathcal{T}_{1}+\mathcal{T}_{2}\right)\right) \leqslant 1
$$

From which one can deduce that

$$
\left\|\mathcal{T}_{1}+\mathcal{T}_{2}\right\|_{\mathbf{b}_{\varphi}^{r, s}}^{(s)} \leqslant N\left(\rho_{1}+\rho_{2}\right) \leqslant N\left(\left\|\mathcal{T}_{1}\right\|_{\mathbf{b}_{\varphi}^{r, s}}^{(s)}+\left\|\mathcal{T}_{2}\right\|_{\mathbf{b}_{\varphi}^{r, s}}^{(s)}+\varepsilon\right)
$$

Since $\varepsilon>0$ is arbitrary, therefore

$$
\left\|\mathcal{T}_{1}+\mathcal{T}_{2}\right\|_{\mathbf{b}_{\varphi}^{r, s}}^{(s)} \leqslant N\left(\left\|\mathcal{T}_{1}\right\|_{\mathbf{b}_{\varphi}^{r, s}}^{(s)}+\left\|\mathcal{T}_{2}\right\|_{\mathbf{b}_{\varphi}^{r, s}}^{(s)}\right) .
$$

Now we shall establish the completeness of $\mathcal{L}_{\mathbf{b}_{\varphi}^{\text {r,s }}}^{(s)}$. Let $\left(\mathcal{T}^{(\mathfrak{i})}\right)$ be a Cauchy sequence in $\mathcal{L}_{\mathbf{b}_{\varphi}^{\mathrm{r}, s}}^{(\mathrm{s})}$, then for $\varepsilon>0$ there exists a positive integer $n_{0}$ such that $\left\|\mathcal{T}^{(i)}-\mathcal{T}^{(j)}\right\|_{\mathbf{b}_{\varphi}^{r, s}}^{(s)}<\varepsilon$ for each $i, j \geqslant n_{0}$. We choose $0<\rho<\varepsilon$ and

$$
\sum_{n=0}^{\infty} \varphi\left(\frac{1}{\rho} \sum_{k=0}^{n} \frac{1}{(s+r)^{n}}\left(\begin{array}{l}
n \\
k
\end{array}\right) s^{n-k} r^{k} s_{k}\left(\mathcal{T}^{(i)}-\mathcal{T}^{(j)}\right)\right) \leqslant 1
$$


for $i, j \geqslant n_{0}$. Using the assumption $\varphi(1)=1$ and the same argument above, one can deduce that $\left\|\mathcal{T}^{(i)}-\mathcal{T}^{(j)}\right\| \rightarrow 0$ as $i, j \rightarrow \infty$. Hence $\left(\mathcal{T}^{(i)}\right)$ is a Cauchy squence in $\mathbf{L}(X, Y)$ and hence converges, say to $\mathcal{T}$, i.e., $\left\|\mathcal{T}^{(i)}-\mathcal{T}\right\| \rightarrow 0$ as $i \rightarrow \infty$. Since $\varphi$ is continuous, therefore using equation (5.1) as $i \rightarrow \infty$,

$$
\sum_{n=0}^{\infty} \varphi\left(\frac{1}{\rho} \sum_{k=0}^{n} \frac{1}{(s+r)^{n}}\left(\begin{array}{l}
n \\
k
\end{array}\right) s^{n-k} r^{k} s_{k}\left(\mathcal{T}^{(i)}-\mathcal{T}\right)\right) \leqslant 1 .
$$

Thus $\mathcal{T} \in \mathcal{L}_{\mathbf{b}_{\varphi}^{r, s}}^{(s)}$ and $\left\|\mathcal{T}^{(i)}-\mathcal{T}\right\|_{\mathbf{b}_{\varphi}^{r, s}}^{(s)} \leqslant \rho<\varepsilon$ as $i \rightarrow \infty$. This establishes the result.

\section{Conclusion}

In this article, we give an upper bound estimation for the norms of Hausdorff matrix and Nörlund matrix as operators from $\ell_{\varphi}$ to $\mathbf{b}_{\varphi}^{r, s}$, thereby obtaining a Hardy type formulae in the case of Hausdorff matrix. We have used Jensen's inequality to prove all the results. Note that by ignoring the weighted version, i.e., by taking $\lambda_{n}=1$ and $v_{n}=1$ for all $n \in \mathbb{N}_{0}$ in the results of Manna [26] and Talebi and Dehgan [37], respectively, then our investigated results in this paper intend to generalize the results obtained by the authors in $[26,37]$. We also defined operator ideal for Orlicz-binomial sequence space and proved its completeness. We expect that the results obtained in this paper might be a reference for further studies in this field.

\section{Acknowledgment}

The research of the first author (T. Yaying) is supported by SERB, DST, New Delhi, India under the grant number EEQ/2019/000082.

\section{References}

[1] P. Z. Alp, E. E. Kara, Some equivalent quasinorms on $\mathrm{L}_{\phi, \mathrm{E}}$, Facta Univ. Ser. Math. Inform., 33 (2018), 739-749. 5

[2] P. Z. Alp, E. E. Kara, The new class $\mathrm{L}_{z, p, \mathrm{E}}$ of s-type operators, AIMS Math., 4 (2019), 779-791.

[3] P. Z. Alp, E. E. Kara, A new class of operator ideals on the block sequence space $\ell_{p}(E)$, Adv. Appl. Math. Sci., 18 (2018), 205-217.

[4] P. Z. Alp, M. Ilkhan, A new class of s-type X(u,v, $\left.\mathrm{l}_{\mathfrak{p}}(\mathrm{E})\right)$ operators, Sakarya University J. Sci., 23 (2019), 792-800. 5

[5] P. Z. Alp, M. İlkhan, On the difference sequence space $l_{p}\left(\tilde{T}^{q}\right)$, Math. Sci. \& Appl. E-Notes, 7 (2019), 161-173. 1

[6] B. Altay, F. Başar, On some Euler sequence spaces of nonabsolute type, Ukr. Math. J., 57 (2005), 1-17. 1

[7] B. Altay, F. Başar, M. Mursaleen, On the Euler sequence spaces which include the spaces $\ell_{p}$ and $\ell_{\infty} I$, Inform. Sci., 176 (2006), 1450-1462. 1, 2

[8] S. Aydin, H. Polat, Difference sequence spaces derived by using Pascal Transform, Fundam. J. Math. Appl., 2 (2019), 56-62. 1

[9] G. Bennet, Factorizing the classical inequalities, Mem. Amer. Math. Soc., 120 (1996). 3

[10] M. C. Bissgin, The binomial sequence spaces of nonabsolute type, J. Inequal. Appl., 2016 (2016), 16 pages. 1

[11] M. C. Bissgin, The binomial sequence spaces which include the spaces $\ell_{p}$ and $\ell_{\infty}$ and geometric properties, J. Inequal. Appl., 2016 (2016), 15 pages. 1, 2

[12] A. Esi, H. Dutta, Some sets of double lacunary invariant sequences defined by four dimensional summable matrices and Orlicz functions, Miskolc Math. Notes, 16 (2015), 805-816. 1

[13] A. Esi, B. Hazarika, A. Esi, New type of lacunary Orlicz difference sequence spaces generated by infinite matrices, Filomat, 30 (2016), 3195-3208. 1

[14] M. Gupta, A. Bhar, Generalized Orlicz-Lorentz sequence spaces and their corresponding operator ideals, Math. Slovaca, 64 (2014), 1475-1496. 5

[15] M. İlkhan, Norms and lower bounds of some matrix operators on Fibonacci weighted difference sequence space, Math. Methods Appl. Sci., 42 (2019), 5143-5153. 1

[16] M. İlkhan, E. E. Kara, A new Banach space defined by Euler totient Matrix operator, Oper. Matrices, 13 (2019), 527-544. 1

[17] A. Jakimovski, B. E. Rhoades, J. Tzimbalario, Hausdorff matrices as bounded operators over $\ell_{\mathrm{p}}$, Math. Z., 138 (1974), 173-181. 3 
[18] G. J. O. Jameson, R. Lashkaripour, Lower bounds of operators on weighted $\ell_{p}$ spaces and Lorentz sequence spaces, Glasgow Math. J., 42 (2000), 211-223. 1

[19] E. E. Kara, M. İlkhan, On a new class of s-type operators, Konuralp J. Math., 3 (2015), 1-11. 5

[20] E. E. Kara, M. Özturk, M. Başarir, Some topological and geometric properties of generalized Euler sequence space, Math. Slovaca, 60 (2010), 385-398. 1

[21] R. Lashkaripour, D. Foroutannia, Inequlity involving upper bounds for certain matrix operators, Proc. Indian Acad. Sci. Math. Sci., 116 (2006), 325-336. 1

[22] R. Lashkaripour, D. Foroutannia, Some inequalities involving upper bounds for some matrix operators I, Czechoslovak Math. J., 57 (2007), 553-572.

[23] R. Lashkaripour, D. Foroutannia, Lower bounds for matrices on weighted sequence spaces, J. Sci. Islam. Repub. Iran., 18 (2007), 49-56.

[24] R. Lashkaripour, G. Talebi, Lower bounds of Copson for Hausdorff matrices on weighted sequence spaces, J. Sci. Islam. Repub. Iran., 22 (2011), 153-157. 1

[25] E. R. Love, Hardy's inequality in Orlicz-type sequence spaces for operators related to generalized Hausdorff matrices, Math. Z., 193 (1986), 481-490. 1, 3, 3.1

[26] A. Manna, Norm inequalities involving upper bounds for certain matrix operators in Orlicz-type sequence spaces, J. Anal., 27 (2019), 761-779. 1, 2, 6

[27] J. Musielak, Orlicz spaces and modular spaces, Springer, Berlin, (1983). 1

[28] M. Mursaleen, K. Raj, Some vector valued sequence spaces of Musielak-Orlicz functions and their operator ideals, Math. Slovaca, 68 (2018), 115-134. 5

[29] S. A. Mohiuddine, K. Raj, Vector valued Orlicz-Lorentz sequence spaces and their operator ideals, J. Nonlinear Sci. Appl., 10 (2017), 338-353.

[30] A. Pietsch, s-numbers of operators in Banach spaces, Studia Math., 51 (1974), 201-223.

[31] A. Pietsch, Operator Ideals, VEB Deutscher Verlag der Wissenschaften, Berlin, (1978).

[32] A. Pietsch, Eigenvalues and s-numbers, Cambridge University Press, New York, (1986).

[33] K. Raj, A. Esi, On some I-convergent sequence spaces over n-normed spaces, J. Anal. Number Theor, 3 (2015), 21-26. 5

[34] H. Roopaei, D. Foroutannia, The norm of matrix operator on Ceśaro weighted sequence spaces, Linear Multilinear Algebra, 67 (2019), 175-185. 1

[35] H. Roopaei, D. Foroutannia, The norms of certain matrix operators from $\ell_{\mathrm{p}}$ spaces into $\ell_{\mathrm{p}}\left(\Delta^{\mathrm{n}}\right)$ spaces, Linear Multilinear Algebra, 67 (2019), 767-776. 1

[36] N. Subramanian, A. Esi, The Nörlund space of double entire sequences, Fasc. Math., (2010), 147-153. 1

[37] G. Talebi, M. A. Dehgan, Upper bounds for the operator norms of Hausdorff matrices and Nörlund matrices on the Euler weighted sequence space, Linear Multilinear Algebra, 62 (2014), 1275-1284. 1, 6

[38] G. Talebi, M. A. Dehgan, Approximation of upper bound for matrix operators on the Fibonacci weighted sequence spaces, Linear Multilinear Algebra, 64 (2016), 196-207. 1

[39] T. Yaying, A. Das, B. Hazarika, P. Baliarshingh, Compactness of binomial difference sequence spaces of fractional order and sequence spaces, Rend. Circ. Mat. Palermo, 68 (2019), 459-476. 1

[40] T. Yaying, B. Hazarika, On sequence spaces generated by binomial difference operator of fractional order, Math. Slovaca, 69 (2019), 901-918. 1 